\title{
Stress Intensity of Debonding for A Rigid Inclusion Near An Angle Dislocation
}

\author{
Xianfeng Wang ${ }^{1, *}$, Feng Xing ${ }^{1}$ and Norio Hasebe ${ }^{2}$ \\ ${ }^{I}$ College of Civil Engineering, Shenzhen University, Shenzhen 518060, PR China \\ ${ }^{2}$ Department of Civil Engineering, Nagoya Institute of Technology, Japan
}

\begin{abstract}
The study of debonding is of importance in providing a good understanding of the bonded interfaces of dissimilar materials. The problem of debonding of an arbitrarily shaped rigid inclusion in an infinite plate with a point dislocation of thin plate bending is investigated in this paper. Herein, the point dislocation is defined with respect to the difference of the plate deflection angle. An analytical solution is obtained by using the complex stress function approach and the rational mapping function technique. In the derivation, the fundamental solutions of the stress boundary value problem are taken as the principal parts of the corresponding stress functions, and through analytical continuation, the problem of obtaining the complementary stress function is reduced to a Riemann-Hilbert problem. Without loss of generality, numerical results are calculated for a square rigid inclusion with a debonding. It is noted that the stress components are singular at the dislocation point, and a stress concentration can be found in the vicinity of the inclusion corner. We also obtain the stress intensity of a debonding in terms of the stress functions. It can be found that when a debonding starts from a corner of the inclusion and extends to another corner progressively, the stress intensity of the debonding increases monotonously; once the debonding extends over the corner points, the value of the stress intensity of the debonding gradually decreases. The relationships between the stress intensity of the debonding and the direction and position of the dislocation are also presented in this paper.
\end{abstract}

Keywords: Debonding, Inclusion, Rational mapping function, Thin plate bending, Dislocation.

\section{INTRODUCTION}

Investigation on the problem of imperfect bonding is important in providing a good understanding of debonding phenomenon and for the validity of the structural assessments. Since Williams [1] first reported the oscillatory near-tip stress behavior of the elastic plane composed of two dissimilar materials, the problems of the interfacial crack and debonding have been discussed by many researchers. However, in most such studies the authors limited themselves to very simple configurations because of the mathematical difficulties arising in analytical solutions. The latter have not, therefore received enough attention in the literature [2-4]. As known, Green's functions that satisfy certain boundary conditions, are very useful in the analysis of the related problems by BEM procedures. The objective in this paper is to derive an analytical solution for the thin plate bending problem with a debonding of an arbitrarily shaped rigid inclusion in an infinite plate with a point dislocation. The formulations of the complex stress function approach given by Savin [5] for the thin plate bending together with the rational mapping function technique ${ }^{6}$ are used in this analysis. Without loss of generality, for the infinite plate with a square rigid inclusion, the stress distribution and the stress intensity of debonding tips are obtained.

*Address correspondence to this author at the College of Civil Engineering, Shenzhen University, Shenzhen 518060, PR China; Tel: +86-755-26534712; Fax: +86-755-26534021; E-mail: xfw@szu.edu.cn

\section{THE RATIONAL MAPPING FUNCTION AND BASIC FORMULA}

Hasebe and Inohara [6] introduced a rational mapping function for relatively complicated configurations. The mapping function is expressed as a finite sum of fractional expressions. It satisfies therefore the basic conditions for a closed form solution. In this study, we consider a square configuration as shown in Fig. (1). With the following rational mapping function,

$z=\omega(\zeta)=E_{0} \zeta+\sum_{k=1}^{2 n} \frac{E_{k}}{\zeta_{k}-\zeta}+E_{-1}$

the exterior of the square inclusion in the $\mathrm{z}$ plane can be transformed onto exterior $\left(\mathrm{S}^{+}\right)$of the unit circle in the $\zeta$ plane. Here $E_{0}, E_{k}$ and $E_{-1}$ are constants, poles $\zeta_{k}$ are located in the unit circle in the $\zeta$ plane. The parameter $\mathrm{n}$ is chosen as 24 in this study.

The complex stress function method ${ }^{5}$ for the elastic thin plate bending problem is employed herein. As it follows from Fig. (1), for the mixed boundary conditions around the rigid inclusion, we denote the bonded boundary by $\mathrm{M}$, and the unbonded boundary by $\mathrm{L}$. The stress boundary condition on $\mathrm{L}$ can be expressed as:

$$
\begin{aligned}
& \kappa \phi(\sigma)+\frac{\omega(\sigma)}{\overline{\omega^{\prime}(\sigma)}} \overline{\phi^{\prime}(\sigma)}+\overline{\psi(\sigma)}=\frac{1}{D(1-v)} \\
& \int_{0}^{s}\left[m(s)+i \int_{0}^{s} p(s) d s\right](d x+i d y)+i C z+C_{1}
\end{aligned}
$$



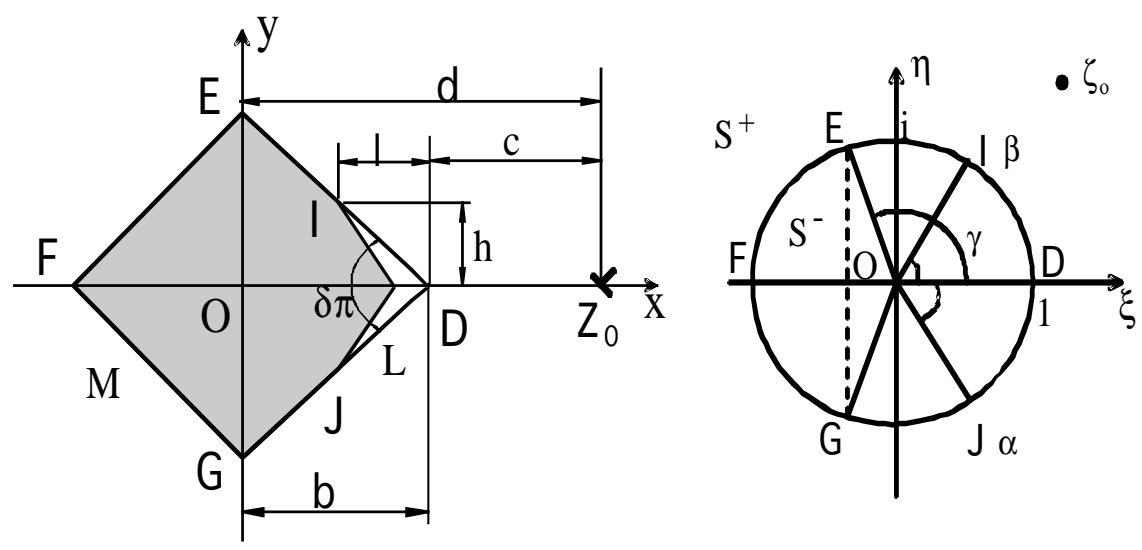

Fig. (1). Infinite plate with a partially bonded inclusion and a point dislocation.

where $v$ is the Poisson ratio of the material, D is the flexural rigidity of the plate $D=E h^{3} /\left[12\left(1-v^{2}\right)\right] . \sigma$ denotes the point on the unit circle corresponding to the boundary point in the z plane, $\kappa=-(3+v) /(1-v), \mathrm{C}$ is a real constant, $C_{1}$ is a complex constant, $\mathrm{m}(\mathrm{s})$ and $\mathrm{p}(\mathrm{s})$ are the bending moment and the bending force per unit length along boundary line L, respectively.

The displacement boundary conditions on $\mathrm{M}$ can be expressed as:

$\phi(\sigma)+\frac{\omega(\sigma)}{\overline{\omega^{\prime}(\sigma)}} \overline{\phi^{\prime}(\sigma)}+\overline{\psi(\sigma)}=\frac{\partial w}{\partial x}+i \frac{\partial w}{\partial y} \quad \sigma \in M$

where $w$ is the deflection of the thin plate.

\section{ANALYSIS OF THE PROBLEM}

As shown in Fig. (1), a rigid inclusion in an infinite plate with a point dislocation has a debonding along its boundary. The solution of this problem represents the Green's function of the corresponding crack problem. Herein, the dislocation of a point in the thin plate bending problem is defined as:

$L=\left\{\frac{\partial w}{\partial x}+i \frac{\partial w}{\partial y}\right\}_{L}$

where \{\}$_{L}$ denotes the increment in value of the expression in the braced brackets when moving around the dislocation point in the counter-clockwise direction. To overcome the difficulty of the described problem, we divide the original formulation into two parts. In part one, a point dislocation is initiated outside a traction free hole in the infinite plate, in which the shape of the hole is the same as that of the inclusion. Part two is complementary, and is stated in such a way that the original mixed boundary condition is satisfied under consideration of stresses and displacements induced by part one. This yields, for each of the stress functions:

$\phi_{G}(\zeta)=\phi_{p 1}(\zeta)+\phi_{p 2}(\zeta), \quad \psi_{G}(\zeta)=\psi_{p 1}(\zeta)+\psi_{p 2}(\zeta)$

where $\phi_{p 2}(\zeta)$ and $\psi_{p 2}(\zeta)$ represent the holomorphic functions defined in $\mathrm{S}^{+}$. The latter denotes the exterior of the unit circle in the $\zeta$ plane.

\section{SOLUTION OF THE STRESS BOUNDARY VALUE PROBLEM}

As shown in Fig. (2), the problem in part one describes a point dislocation initiated outside a traction free hole in the infinite plate. This is a stress-type boundary value problem. The stress functions to be obtained are comprised of two parts as follows:

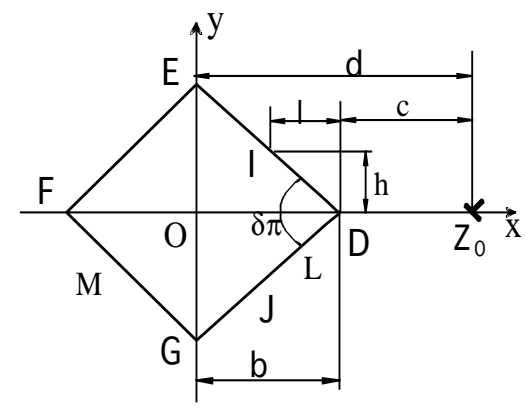

Fig. (2). Stress boundary value problem.

$$
\phi_{p 1}(\zeta)=\phi_{a}(\zeta)+\phi_{b}(\zeta), \quad \psi_{p 1}(\zeta)=\psi_{a}(\zeta)+\psi_{b}(\zeta)
$$

where $\phi_{a}(\zeta)$ and $\psi_{a}(\zeta)$ represent the irregular, multi-valued terms. They can be expressed as:

$$
\begin{aligned}
& \phi_{a}(\zeta)=\frac{L}{2 \pi i} \frac{1-v}{4} \log \left(\zeta-\zeta_{o}\right) \\
& \psi_{a}(\zeta)=\frac{\bar{L}}{2 \pi i} \frac{3+v}{4} \log \left(\zeta-\zeta_{o}\right)-\frac{L}{2 \pi i} \frac{1-v}{4} \frac{\overline{\omega\left(\zeta_{o}\right)}}{\omega^{\prime}\left(\zeta_{o}\right)\left(\zeta-\zeta_{o}\right)}
\end{aligned}
$$

where $\zeta_{0}$ denotes the point in the $\zeta$ plane where the dislocation is applied. $\phi_{b}(\zeta), \psi_{b}(\zeta)$ represent the regular terms. Using the traction free boundary condition of (2), analytical continuation can be accomplished over the boundary. Then $\psi_{p 1}(\zeta)$ can be represented in terms of $\phi_{p 1}(\zeta)$ in the entire region:

$$
\psi_{p 1}(\zeta)=-\kappa \overline{\phi_{p 1}(1 / \bar{\zeta})}-\frac{\overline{\omega(1 / \bar{\zeta})}}{\omega^{\prime}(\zeta)} \phi_{p 1}^{\prime}(\zeta) \quad\left(\zeta \in S^{+}\right)
$$


We substitute (6) into this expression. Since $\psi_{b}(\zeta)$ is a regular function in $\mathrm{S}^{+}$, function $\bar{\phi}_{b}(1 / \zeta)$ can be determined by removing the irregular terms defined in $\mathrm{S}^{+}$. Then by the inverse transformation, $\phi_{b}(\zeta)$, which should be continuous over the boundary when the surface is traction free, can be determined as:

$$
\begin{aligned}
& \phi_{b}(\zeta)=\sum_{k=1}^{2 n}\left[-\frac{\bar{L}}{2 \pi i} \frac{1-v}{4} \frac{1}{\left(\bar{\zeta}_{k}^{\prime}-\bar{\zeta}_{o}\right)}+\overline{A_{b k}}\right] \cdot \frac{B_{k}}{\kappa\left(\zeta-\zeta_{k}\right)} \\
& -\frac{\bar{L}}{2 \pi i} \frac{1-v}{4 \kappa} \cdot \frac{\omega\left(\zeta_{o}\right)-\omega\left(\zeta_{o}^{\prime}\right)}{\overline{\omega^{\prime}\left(\zeta_{o}\right)}} \cdot \frac{\zeta_{o}^{\prime 2}}{\zeta_{o}^{\prime}-\zeta}+\frac{L}{2 \pi i} \frac{3+v}{4 \kappa} \log \left(\frac{1}{\zeta}-\bar{\zeta}_{o}\right) \quad\left(\zeta \in S^{+}\right)
\end{aligned}
$$

where we denote $\zeta_{k}^{\prime} \equiv 1 / \bar{\zeta}_{k}, A_{b k} \equiv \phi_{b}^{\prime}\left(\zeta_{k}^{\prime}\right), \quad \zeta_{o}^{\prime} \equiv 1 / \bar{\zeta}_{o}$ and $B_{k} \equiv E_{k} / \overline{\omega^{\prime}\left(\zeta_{k}^{\prime}\right)}$. The undetermined parameters $A_{b k}$ can be obtained by letting $\zeta=\zeta_{k}^{\prime}(\mathrm{k}=1,2, \ldots, 2 \mathrm{n})$ individually in $\phi_{b}^{\prime}(\zeta)$, a series of algebraic equations are obtainable, from which the real and imaginary parts of $A_{b k}$ and $\bar{A}_{b k}$ can be determined, while $\psi_{p 1}(\zeta)$ can be determined by (8).

\section{SOLUTION OF THE INCLUSION DEBONDING PROBLEM}

In the previous section, the solution of part one has been obtained. In fact, it represents the Green's function of the stress boundary value problem. For the problem of a point dislocation applied to an infinite plate with the partially bonded inclusion, which is shown in Fig. (1), the stress functions $\phi_{p 2}(\zeta)$ and $\psi_{p 2}(\zeta)$ of the complementary parts in (5) should be determined. Similar to (8), the formulae of analytical continuation is used in this section, so that $\psi_{G}(\zeta)$ is expressed by $\phi_{G}(\zeta)$ in the entire region. Substituting the particular expressions of stress functions $\phi_{G}(\zeta)$ and $\psi_{G}(\zeta)$ into boundary conditions (2) and (3), the following expressions can be obtained:

$\phi_{p 2}^{+}(\sigma)-\phi_{p 2}^{-}(\sigma)=0 \quad \sigma \in L$

$\phi_{p 2}^{+}(\sigma)-\kappa \phi_{p 2}^{-}(\sigma)=(\kappa-1) \phi_{p 1}(\sigma) \quad \sigma \in M$

where the superscripts ${ }^{+}$and ${ }^{-}$indicate the limit values of the function $\phi_{p 2}(\zeta)$ as it approaches the boundary from the regions $S^{+}$and $S^{-}$, respectively. $\phi_{p 1}(\zeta)$ can be obtained by (6), (7) and (9). We found that the determination of $\phi_{p 2}(\zeta)$ in (10a) and (10b) represents a Riemann-Hilbert problem, of which the solution is expressed in the following form [7]:

$\phi_{p 2}(\zeta)=\frac{\chi(\zeta)}{2 \pi i} \int_{M} \frac{(\kappa-1) \cdot \phi_{p 1}(\sigma)}{\chi^{+}(\sigma)(\sigma-\zeta)} d \sigma+\chi(\zeta) \cdot G_{1}(\zeta)$

The integral term in (11) can be obtained by replacing the single-path integral with a contour integral. The latter can be evaluated through the residues [8]. This yields:

$$
\begin{aligned}
& \phi_{p 2}(\zeta)=-\frac{L}{2 \pi i} \frac{1-v}{4}\left[\ln \left(\zeta-\zeta_{0}\right)+\chi(\zeta) \int_{\zeta_{o}}^{\infty} \frac{1}{\chi(\sigma)(\sigma-\zeta)} d \sigma\right] \\
& +\frac{L}{2 \pi i} \frac{1-v}{4}\left[\ln \left(1 / \zeta-\bar{\zeta}_{o}\right)+\chi(\zeta) \int_{\zeta_{o}^{\prime}}^{0} \frac{1}{\chi(\sigma)(\sigma-\zeta)} d \sigma\right] \\
& -\frac{\bar{L}}{2 \pi i} \frac{(1-v)}{4 \kappa} \cdot \frac{\left[\omega\left(\zeta_{o}\right)-\omega\left(\zeta_{o}^{\prime}\right)\right]}{\omega^{\prime}\left(\zeta_{o}\right)} \cdot\left[1-\frac{\chi(\zeta)}{\chi\left(\zeta_{o}^{\prime}\right)}\right] \frac{\zeta_{o}^{\prime 2}}{\left(\zeta-\zeta_{o}^{\prime}\right)} \\
& -\frac{1}{\kappa} \sum_{k}\left[-\frac{\bar{L}}{2 \pi i} \frac{1-v}{4} \frac{1}{\left(\overline{\zeta^{\prime}}{ }_{k}-\bar{\zeta}\right)}+\overline{A_{b k}}\right] \cdot\left[1-\frac{\chi(\zeta)}{\chi\left(\zeta_{k}\right)}\right] \frac{B_{k}}{\left(\zeta-\zeta_{k}\right)} \\
& +\chi(\zeta) \cdot G_{1}(\zeta)
\end{aligned}
$$

where Plemelj function can be expressed as

$$
\begin{aligned}
& \chi(\zeta)=(\zeta-\alpha)^{m}(\zeta-\beta)^{1-m} \\
& m=0.5+i \frac{\log |\kappa|}{2 \pi}
\end{aligned}
$$

with $\alpha$ and $\beta$ being the points on the unit circle corresponding to the tips of debonding as shown in Fig. (1). Since $\chi(\zeta)$ is a Plemelj function, the branch of $\lim _{\zeta \rightarrow \infty}[\chi(\zeta) / \zeta]=1$ is selected.

In the expression for $\phi_{p 2}(\zeta)$, there still exist integral terms as shown in (12), while their first derivatives are expressed in the analytical form containing no integrals [8]. Function $G_{1}(\zeta)$ in (12) is rational throughout $S^{+}$. Upon exploiting the formulae of analytical continuation, and keeping in mind that $\phi_{p 1}(\zeta)$ has satisfied the relationship (8), we obtain,

$G_{1}(\zeta)=\sum_{k=1}^{2 n} \frac{\bar{A}_{2 k} B_{k}}{\kappa \chi\left(\zeta_{k}\right)\left(\zeta-\zeta_{k}\right)}$

where $A_{2 k} \equiv \phi_{p 2}^{\prime}\left(\zeta_{k}^{\prime}\right)$ and $B_{k} \equiv E_{k} / \overline{\omega^{\prime}\left(\zeta_{k}^{\prime}\right)}, A_{2 k}$ can be obtained similarly to the previous section. That is, letting $\zeta=\zeta_{k}^{\prime}(\mathrm{k}=1,2, \ldots, 2 \mathrm{n})$ individually in its first derivative $\phi_{p 2}^{\prime}(\zeta)$, one reduces the problem to a system of linear algebraic equations.

\section{THE STRESS INTENSITIES OF DEBONDING TIPS}

We have known that at the vicinity of the debonding tips $\mathrm{I}$ and $\mathrm{J}$ shown in Fig. (1), there is a highly oscillatory stress field. Hasebe et al., [9] studied a branching problem for a crack, in which the debonding was generated at the end of a displacement constraint of a thin plate subjected to the outof-plane loading, and a definition of the stress intensity of debonding was proposed. For the debonding tip $\mathrm{J}$ ( $\alpha$ in the $\zeta$ plane shown in Fig. 1), the stress intensity of debonding $\Omega$ is: 


$$
\begin{aligned}
& \Omega=\Omega_{1}+i \Omega_{2}=2 \sqrt{2} D(1+v) \cdot e^{-\pi \delta} \lim _{z \rightarrow z_{j}}\left(z-z_{j}\right)^{1-m} \phi^{\prime}(z) \\
& =2 \sqrt{2} D(1+v) \cdot e^{-\pi \delta} \frac{\left\{\omega^{\prime}(\alpha)(\alpha-\beta)\right\}^{1-m}}{\omega^{\prime}(\alpha)(\alpha-\beta)} g(\alpha)
\end{aligned}
$$

where $\delta=\log |\kappa| / 2 \pi$, the stress function $\phi^{\prime}(\zeta)$ can be expressed in the following form:

$\phi^{\prime}(\zeta)=(\zeta-\alpha)^{m-1}(\zeta-\beta)^{-m} g(\zeta)+g_{0}(\zeta)$

where $g_{0}(\zeta)$ is a regular function. At the vicinity of the debonding tip on the interface, we found that the bending and twisting moment components can be expressed in terms of the absolute value $\left|\Omega_{J}\right|$, rather the complex value $\Omega_{\mathrm{J}}$. Therefore, we use $\left|\Omega_{J}\right|$ as the index of the stress intensity of debonding. This is equivalent to that of the strain energy release rate for the evaluation of the debonding [9].

\section{NUMERICAL EXAMPLES AND DISCUSSION}

To demonstrate the effectiveness of the proposed solution, numerical computations are carried out for the problem with the square inclusion. For the sake of convenience, the non-dimensional stress intensity of debonding at tip $\mathrm{J}$ is used as follows:

$$
F_{J}=\frac{v+3}{v+1} \frac{\sqrt{b}}{D}\left|\Omega_{J}\right|
$$

where $b$ is the scale of the square hole as shown in Fig. (1).

The stress distributions along the $\mathrm{x}$-axis for the plate subjected to a point dislocation are depicted in Figs. (3 and 4$)$ for $\mathrm{L}=1$ and $\mathrm{L}=i$ respectively. The following parameters are chosen for the actual computing: inclusion debonding length $1 / 2 b=0.654$, Poission ratio $v=0.25$ and the dislocation is initiated at $(3 b, 0)$. It is worth noting that the stress levels at the vicinity of the dislocation point increase significantly, while decrease gradually to zero as the value of $x$ approaches infinity. At the corner of the point $(b, 0)$ in Fig. (1), a stress

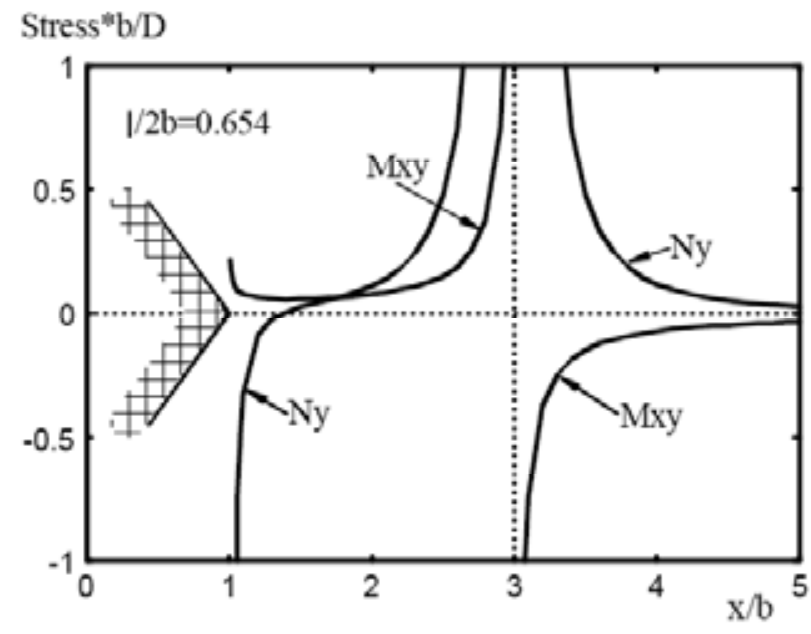

Fig. (3). Stress distribution on the $x$-axis ( $L$ is at $(3 b, 0), L=1$, $v=0.25)$. concentration can be found. Fig. (5) shows the variation of the non-dimensional stress intensity of debonding at tip $\mathrm{J}$ when the directional angle $\theta(\mathrm{L}=\cos \theta+\mathrm{i} \sin \theta)$ of a point dislocation rotates ninety degrees for two different debonding lengths $1 / 2 b=0.367,1 / 2 b=0.654$. Fig. (6) shows the nondimensional stress intensity of debonding at tip $\mathrm{J}$ versus the dislocation position on the $\mathrm{x}$-axis also for two different debonding lengths $1 / 2 b=0.367,1 / 2 b=0.654$. Fig. (7) shows the non-dimensional stress intensity of debonding at tip $\mathrm{J}$ versus the debonding length $1 / 2 \mathrm{~b}$ for three Poisson ratios $0.0,0.25$ and 0.5 . It should be noted that manifest high trend of $F_{J}$ can be found at the vicinity of the inclusion corner $G(1 / 2 b=0.5)$.

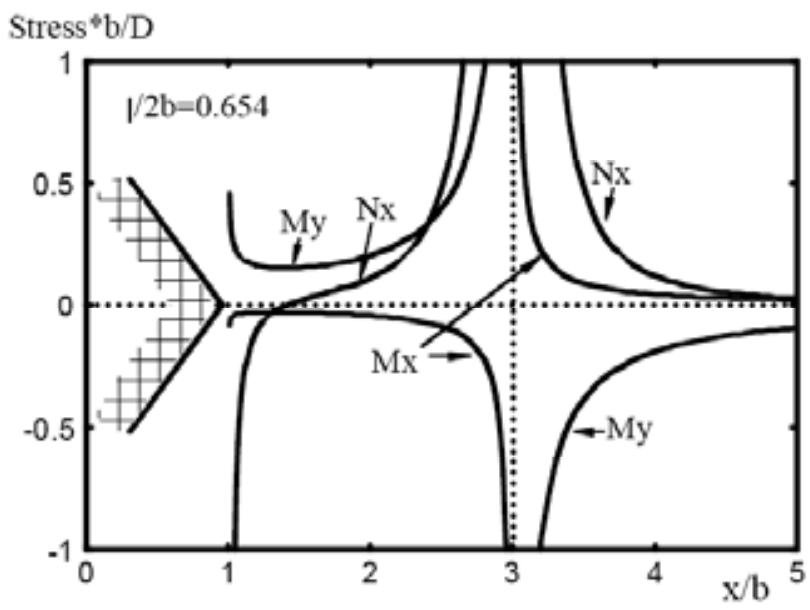

Fig. (4). Stress distribution on the $x$-axis ( $L$ is at $(3 b, 0), L=i, v$ $=0.25)$.

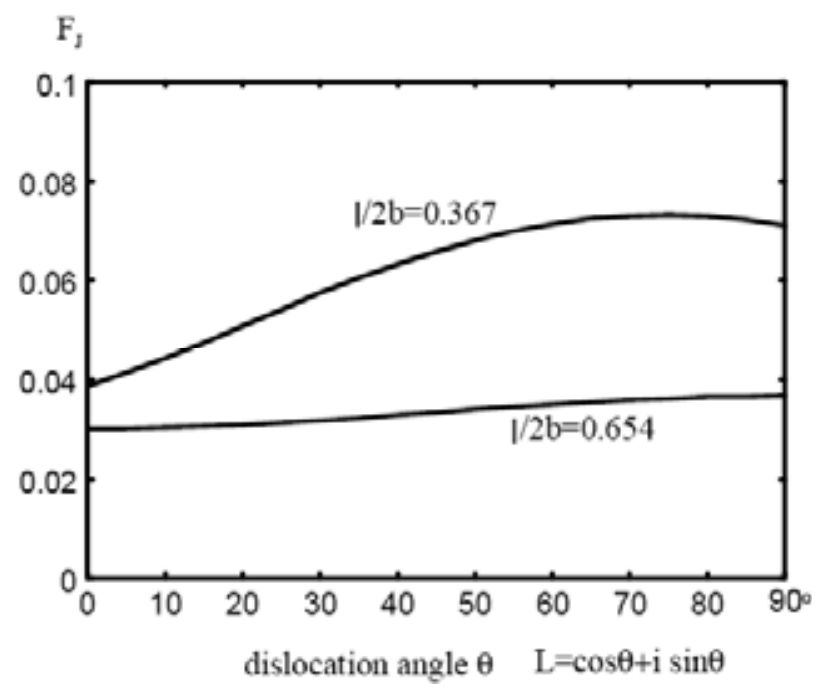

Fig. (5). Non-dimensional stress intensity of debonding $F_{J}$ versus dislocation angle.

Hence, we have obtained the closed form solutions for the Green's functions of point dislocation under the mixed boundary conditions for the thin plate bending problem. The solution of the mixed boundary value problem is obtained by the use of the solution of the stress boundary value problem 


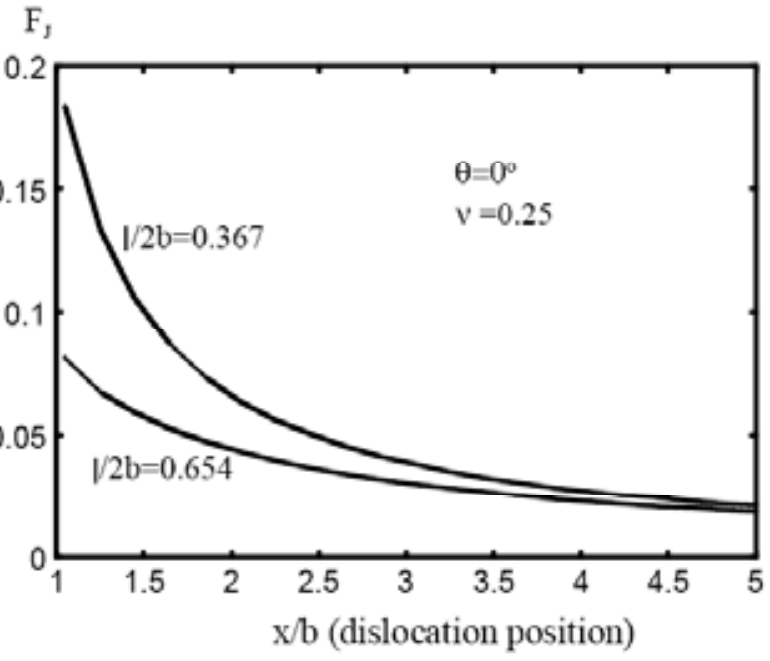

Fig. (6). Non-dimensional stress intensity of debonding $F_{J}$ versus dislocation position.

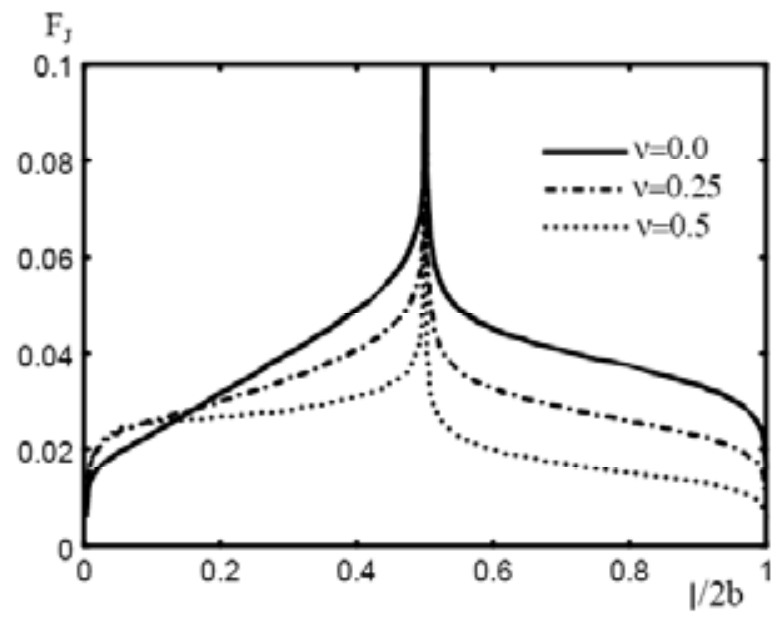

Fig. (7). Non-dimensional stress intensity of debonding $F_{J}$ versus debonding length.

as the principal part in the original stress functions. This Green's function could be used in analyzing a variety of problems (the crack problem in thin plate bending). And it can also be used as a kernel of boundary integral equations in BEM analysis, where it simplifies the calculation of the boundary integrals along the inclusion [10]. In addition, this study proves that the rational mapping function technique together with the complex stress function's approach is particularly effective in solving inclusion problems of intricate configurations. In general, this method is applicable to arbitrary shape, and the closed solution for the relevant problem can be obtained for a simply connected region.

\section{CONCLUSIONS}

The Green's function of a point dislocation was derived for the mixed boundary value problem of an infinite plate with a partially bonded rigid inclusion. The stress functions are obtained in closed form. The key points in the procedure are the utilization of the Green's function of an appropriate stress boundary value problem and the rational mapping technique. This is particularly effective in solving inclusion problems of intricate configurations. The stress intensities of debonding tips were computed. This Green's function can also be used as a kernel for boundary integral representations in BEM analysis, where it can notably simplify the procedure of the standard boundary integral equations.

\section{ACKNOWLEDGEMENTS}

The authors gratefully acknowledge the financial support provided by the Natural Science Foundation for the National Science Fund for Distinguished Young Scholars (No. 50925829), the National Natural Science Foundation of China (No.51078238), the Science and Technology Foundation of Shenzhen City (JC201005280571A) and the Science and Technology Foundation of Shenzhen University (No.000015).

\section{CONFLICT OF INTEREST}

None declared.

\section{REFERENCES}

[1] M. L. Williams, "The stress around a fault or crack in dissimilar media", Bull. Seismol. Soc. Am., vol. 49, pp. 199-204, 1959.

[2] J. R. Rice. "Elastic fracture mechanics concepts for interfacial cracks", J. Appl. Mech., ASME, vol. 55, pp. 98-103, 1988.

[3] M. Comninou, "The interface crack", J. Appl. Mech., ASME, vol. 44, pp. 631-636, 1977.

[4] M. Comninou, "An overview of interface crack", Eng. Fract. Mech., vol. 37, pp. 197-208, 1990.

[5] G. N. Savin, Stress Concentration Around Holes, 1st ed. Eugene Gros, Translator: Pergamon Press, Oxford, 1961.

[6] N. Hasebe, S. Inohara, "Stress analysis of a semi-infinite plate with an oblique edge crack", Ingenieur-Archiv, vol. 49, pp.51-62, 1980.

[7] N. I. Muskhelishvili, Some Basic Problems of the Mathematical Theory of Elasticity, Noordhoff Ltd., Groningen, The Netherlands, 1963.

[8] N. Hasebe, H. Irikura, and T. Nakamura, "A solution of the mixed boundary condition value problem for an infinite plate with a hole under uniform heat flux", J. Appl. Mech., ASME, vol. 58, pp. 9961000, 1991.

[9] N. Hasebe, M. Miwa, and M. Nakashima, "Branching problem of a crack and a debonding at the end of a clamped edge of thin plate", in Proc. $9^{\text {th }}$ Int. Conf. Fracture, Sydney, Australia, 1997, pp. 22192226.

[10] Y. A. Melnikov, "Some applications of the Green's function method in mechanics", Int. J. Solids Struct., vol. 13, pp. 10451058, 1977. 